\title{
Promoting awareness of fetal alcohol spectrum disorder among health professionals and the public through nursing faculty champions
}

\author{
Lili Ma ${ }^{1}$, Kathryn Puskar ${ }^{2}$, Irene Kane ${ }^{2}$, Emily Knapp ${ }^{2}$, Ann M. Mitchell ${ }^{* 2}$ \\ ${ }^{1}$ Capital Medical University, School of Nursing, Beijing, China \\ ${ }^{2}$ School of Nursing, University of Pittsburgh, Pittsburgh, PA, United States
}

Received: August 7, 2017

DOI: $10.5430 / j n e p . v 8 n 2 p 125$

\author{
Accepted: October 16, 2017 \\ Online Published: October 24, 2017 \\ URL: https://doi.org/10.5430/jnep.v8n2p125
}

\begin{abstract}
Fetal alcohol spectrum disorder (FASD) affects all communities and is an underestimated problem worldwide and in China. FASD is the most common preventable cause of intellectual disabilities and behavior problems. However, prevention efforts require knowledge about FASD, importantly, the education of health professionals who communicate that knowledge to the public during care administered in diverse practice settings. Implementing a nursing faculty champions (charismatic advocates for FASD prevention belief, practice, program, policy and/or technology) model to advocate for educating Chinese nurses, nursing students, other health professionals, and the public about FASD is a logical, quality-driven, healthcare action. The actions undertaken by one nurse champion, a Capital Medical University Chinese professor, to promote FASD awareness among Chinese health professionals and the public population will be presented. Through this faculty nurse champion, thousands of Chinese health providers and public citizens were educated regarding FASD. Planned next steps include enrolling more Chinese nursing faculty champions, developing nursing curricula at Capital Medical University, and increasing research attention on FASD. Nurse faculty champions are an effective and practical method to promote FASD awareness among Chinese health professionals and the public.
\end{abstract}

Key Words: Champion, Fetal Alcohol Spectrum Disorder, Intervention, Prenatal Alcohol Exposure, Prevention

\section{INTRODUCTION}

Fetal alcohol spectrum disorder (FASD) is an umbrella term for a range of birth defects caused by prenatal exposure to ethyl alcohol. ${ }^{[1]}$ FASD is the most common preventable cause of intellectual disabilities and behavior problems. ${ }^{[1]}$ However, prevention efforts require educated health professionals who communicate FASD knowledge to the public during care provided in diverse practice settings. Educating nurses, the most trusted group of healthcare professionals, ${ }^{[2]}$ is important because nurses can play a critical role in the success of FASD prevention, intervention, and treatment as they care for patients. Further, nurses can lead the research progress needed to discover additional means to address the lifelong consequences of FASD. Therefore, implementing a nursing faculty champion model to advocate for educating nurses, nursing students, other health professionals, and the public about FASD is a logical, quality-driven health action. This paper will review FASD, FASD in China, and the importance of its prevention through a creative nurse champion role.

\subsection{What is FASD?}

FASD encompasses several conditions depending on the type of symptoms: fetal alcohol syndrome (FAS), partial fetal alcohol syndrome (pFAS), alcohol-related neurodevelopmental disorder (ARND), and alcohol-related birth defects

*Correspondence: Ann M. Mitchell; Email: ammi@pitt.edu; Address: School of Nursing, University of Pittsburgh, Pittsburgh, PA, United States. 
(ARBD). ${ }^{[3]}$

FAS represents the most widely known diagnosis in the spectrum (FASD). Children with FAS might have abnormal facial features (e.g. short palpebral fissures, smooth philtrum, and a thin vermilion border of the upper lip), prenatal and postnatal growth retardation, and functional and structural central nervous system (CNS) abnormalities (e.g. intellectual disabilities).$^{[4]}$ The partial fetal alcohol syndrome (pFAS), represents a child who does not meet the full diagnostic criteria for FAS, but has a history of prenatal alcohol exposure, some of the facial abnormalities, as well as a growth problem or CNS abnormalities. ${ }^{[5]}$ The child with ARND might exhibit intellectual disabilities and problems with behavior and learning, whereas the child with ARBD might manifest problems with the heart, kidneys, and/or bones, as well as with hearing and/or vision. ${ }^{[5]}$ The neurobehavioral disorder associated with prenatal alcohol exposure (ND-PAE) is a newly proposed mental health diagnosis associated with the teratogenic effects of in-utero exposure to alcohol. ${ }^{[6]}$ This diagnosis defines more precisely the developmental and behavioral manifestations associated with prenatal exposure.

The medical, educational, and other services required to care for an individual with FASD, and their family, results in a large economic burden. In the 1980s, the estimated annual FAS-related expenses for the United States increased from $\$ 75$ million to $\$ 4$ billion, with the lifetime cost of care approaching $\$ 1.4$ million. $^{[7]}$ However, the economic burden may be outweighed by the emotional burden faced by the child's family, caregivers, and communities.

\subsection{Cause and prevention}

The direct cause of FASD is the consumption of alcohol by the mother-to-be. When a pregnant woman drinks alcohol, the alcohol in her blood can pass to her baby through the umbilical cord, which may damage the developing fetus. There is no known safe quantity, frequency, type, or timing of alcohol consumption during pregnancy, but having no prenatal alcohol exposure translates into no FASD. ${ }^{[3]}$

Because FASD is the most common preventable cause of intellectual disabilities in the world, efforts at prevention are paramount. To prevent FASD, a woman should not drink alcohol while she is pregnant, or if she is not using contraception and might become pregnant. Hence, it is critical to provide women of childbearing age appropriate education regarding alcohol consumption.

\subsection{Epidemiology of alcohol consumption in China}

Alcohol production and drinking is deeply rooted in Chinese history and culture. Drinking is socially accepted and plays a considerable role in major events in Chinese festivals, such as Chinese New Year, weddings, and birthdays. Furthermore, in recent decades, ${ }^{[8]}$ alcohol consumption in China has been rising faster than in any other part of the world, which could potentially be linked to active marketing by the alcohol industry, and increased income. ${ }^{[8]}$ According to the 2014 Global Status Report on Alcohol and Health, ${ }^{[9]}$ the average alcohol per capita consumption (in liters of pure alcohol) by people over 15 years between 2008 and 2010 was 6.7. Types of alcoholic beverages included: $69 \%$ in spirits, $28 \%$ in beer, and $3 \%$ in wine.

It is important to note that drinking alcohol in China is a gender-related issue, as men tend to drink more than women. According to one investigation, ${ }^{[10]} 55.6 \%$ of men and $15 \%$ of women reportedly drank alcohol. Among those current drinkers, $62.7 \%$ of men and $51.0 \%$ of women reported excessive drinking (daily consumption of pure alcohol more than $25 \mathrm{~g}$ for men and more than $15 \mathrm{~g}$ for women), $26.3 \%$ and $7.8 \%$, respectively reported frequent drinking (drinking 5 to 7 days per week), and $57.3 \%$ and $26.6 \%$, respectively reported binge drinking (consuming $50 \mathrm{~g}$ or more pure alcohol for men and $40 \mathrm{~g}$ or more for women on at least 1 day in the previous 12 months). ${ }^{[11]}$ The different alcohol drinking patterns between men and women in China are related to the traditional Chinese culture, which generally discourages women from drinking.

However, this traditional trend is diminishing with the development of the economy, the parallel increasing income of Chinese women, and the culture globalization. Moreover, it is important to note that Chinese women may deny their drinking, especially drinking during pregnancy, because of historical social pressures, including gender expectations, as well as motherhood expectations. ${ }^{[4]}$ Another research study reports that $10 \%$ of pregnant women in urban China and $6.2 \%$ of pregnant women in rural China drink alcohol. ${ }^{[12]}$

\subsection{FASD in China}

FASD affects all communities and is an underestimated problem worldwide. ${ }^{[13]}$ Although worldwide healthcare providers have been aware of the correlation between birth defects and prenatal alcohol exposure for over 40 years, there is limited information regarding individuals with FASD in China because of insufficient research in this area. According to a systematic review report in 2017, Wang and D'Amato reported FASD cases are very rare in China. ${ }^{[4]}$ However, the prevalence rates of these disorders may be underestimated because health professionals in China lack FASD knowledge and thus are unaware of the FASD span of symptomatology. Currently in China, there is a lack of accurate, routine screening in prenatal clinics and pediatric settings, and no universal suitable diagnosis for individuals with FASD. ${ }^{[4]}$

ISSN 1925-4040 E-ISSN 1925-4059 


\section{MeTHODS}

\subsection{Promoting awareness of FASD}

To eliminate this discrepancy, it is imperative to provide health professionals appropriate education regarding alcohol consumption and alcohol screening. ${ }^{[14]}$ Only if Chinese healthcare providers are well-informed and knowledgeable about providing evidence-based screening and subsequent patient education will Chinese women of childbearing age be appropriately educated on the risks associated with prenatal alcohol consumption and prevention of FASD for their unborn child.

The University of Pittsburgh, School of Nursing, in collaboration with the University of Alaska Anchorage, and the University of California San Diego, has developed a number of definitions related to nurse champions. As part of their work they have identified different types of FASD nurse champions: Executive Champions, Nurse Leader Champions, Faculty Champions, and Student Nurse Champions. The Executive Champions are healthcare administrators who advocate for the implementation of Alcohol Screening and Brief Intervention (alcohol SBI) for FASD prevention in organizations, healthcare systems, and communities. Nurse Leader Champions are those who advocate for and promote alcohol SBI for FASD prevention and skill development in their respective clinical settings including in diverse clinical settings; Faculty Nurse Champions are those who advocate for and promote alcohol SBI for FASD prevention and skill development in their professional educational systems and in continuing education venues; and Student Nurse Champions are those students and recent graduates who advocate for alcohol SBI and FASD prevention in their classes, as well as in their future places of employment. The role of any champion can result in both short- and long-term outcomes, which include increased understanding regarding the benefits of alcohol screening and brief interventions for the prevention of FASD and an increase in the number of healthcare professionals who include alcohol SBI into their clinical practice. The purpose of this project was the development and education of a Chinese Nurse Faculty Champion to collaborate with the Capital Medical University, School of Nursing in Beijing to promote FASD awareness and alcohol SBI education in China.

\subsection{Nurse faculty champion implementation}

To achieve the project purpose, the Chinese nurse faculty champion actively attained alcohol SBI and FASD education within the research team, and systematically reviewed international, including Chinese literature on FASD. Next, she designed an FASD infographic in Mandarin Chinese, as well as developed extended Chinese text information for expanded healthcare professional education. Upon completion of educational materials, the faculty champion: 1) disseminated the infographic by posting paper-based copies on billboards in busy places of Capital Medical University and University associated hospitals; 2) distributed leaflets to the nursing stations of the hospitals; and 3) promoted the Chinese infographic along with extended text information through a self-developed WeChat public account.

\subsection{Partnership and nurse faculty champion action}

The Capital Medical University School of Nursing (CMUSON) and the University of Pittsburgh, School of Nursing have mutually approved and organized a nursing faculty Visiting Scholar Program. The CMUSON, Nurse Faculty Champion, a visiting scholar who was assigned at the University of University of Pittsburgh, School of Nursing (UPSON), was sponsored by the Education Bureau of Beijing, China. The visiting CUMSON faculty scholar participated in research activities at the University of Pittsburgh, School of Nursing on alcohol SBI and FASD prevention for six months. Participation in the School's FASD prevention grant activities included collaboration with and consent from the University of Alaska Anchorage, and the University of California San Diego. Biweekly grant meetings, review of PowerPoint presentations made by the grant team on alcohol SBI and FASD, and a visit to the Pittsburgh, Pennsylvania-based Institute for Research, Education and Training in Addictions (IRETA) allowed the scholar to review alcohol education, training activities, and programs related to substance use. The Champion also participated in training webinars on FASD including: Screening, Assessment, and Diagnosis; Neurobehavioral Disorders associated with Prenatal Alcohol Exposure; and Treatment across the Lifespan for Individuals with FASD which complimented the educational experience. The CMUSON faculty scholar's knowledge gains provided intense motivation to make a difference in her country of origin, and her dedication to patient care led to her request to become a Faculty Nurse Champion with the help of the FASD grant-funded team. Discussions with the team and the visiting scholar's faculties at her School of Nursing resulted in the development of an FASD prevention project for feasible implementation in China.

\subsection{The development of Chinese infographic and text in- formation}

The first step in the preparation of the Chinese infographic and the extended text information was to review the updated literature pertaining to FASD internationally and specifically for China. English research articles were reviewed, as well as research articles published in Mandarin Chinese, which included a systematic search of the largest Chinese National 
Knowledge Infrastructure (CNKI) and the Wanfang database databases reported the earliest studies and continue to hold to summarize all the research and articles identified as re- the most recently published studies. lating to alcohol consumption and FASD in China. These

\section{ALCOHOL AND PREGNANCY? \\ Understanding the facts for a healthier future for you and your baby}

\section{IS IT OK TO DRINK BEER, WINE, OR LIQUOR \\ WHILE PREGNANT?}

No. There is no known safe amount of alcohol to drink during pregnancy or while trying to get pregnant. There is also no known safe time to drink while pregnant.

Drinking beer, wine, or liquor while pregnant may harm the development of your baby, causing life-long challenges.

\section{2

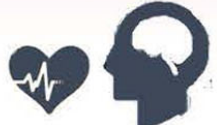

Problems with the heart, brain, and other organ development
Learning disabilities, poor memory, and difficulties paying attention

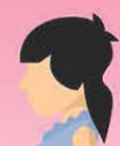

!

Fetal Alcohol Spectrum Disorder (FASD)

Drinking alcoholic beverages while pregnant may interfere with your baby's development, causing physical defects, and potentially leading to life-long behavioral and learning problems.

\section{DRINKING IN CHINA}

$15 \%$ of Chinese women drink alcohol (1)

$2.5 \%$ women drinkers drink alcohol heavily (1).

$10 \%$ pregnant women in urban China drink alcohol and $6.2 \%$ pregnant women in rural China drink alcohol (2)

Few FASD cases have been reported due to insufficient attention and evidenced-based knowledge such as universal suitable diagnosis for individual with FASD given the various country-based levels of understanding (3).

Many women do not plan to become pregnant and often times do not know they are pregnant until 4-6 weeks.

A woman may be drinking and not even know she is pregnant. To prevent alcohol-exposed pregnancies, it is important to stop drinking if there is a chance you may be pregnant.

\section{Reference}

(1) World Health Organization(WHO).(2014) Global status report on alcohol and health 2014.

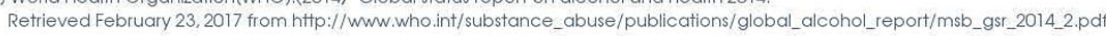

(2) Ren, A., Zhang, L., Hao, L., Li. Z., \& Tian, Y. (2007). Comparison of blood folate levels among pregnant Chinese women in areas with high and low prevalence of neural tube defects. Public Health Nutrition, 10(8), 762-768

Retrieved February 23. 2017 from https://www.cambridge.org/core/journals/public-health-nutrition/article/div-classtitlecomparison-of-blood-folate-levelsamong-pregnant-chinese-women-in-areas-with-high-and-low-prevalence-of-neural-tube-defectsdiv/F99CA 1B620CF834E7B401B121B034332

(3) Wang, Y.Y., D'Amato, R.C. (2017). Understanding fetal alcohol spectrum disorders in China. J Pediatr Neuropsychol. Retrieved February 23, 2017 from http://link.springer.com/article/10.1007/s40817-017-0029-0

Ths project was supported in part by funds from the U.S. Department of Health and Human Senvices, Centers tor Disease Control and Prevention, under grant number 1 U84DD001135. The information Thk post
}

Figure 1. FASD infographic in English 
Based on the CMUSON faculty nurse champion knowledge gains on FASD, the first version of infographic was developed in English. The English version of the infographic was sent to the grant team members for review and discussion in the grant meeting. The team members gave suggestions and comments on the first version of the infographic and it was revised accordingly. After the English version of the infographic had been agreed upon by all team members, the visiting scholar translated it into Mandarin Chinese, the official language in China. Images on the infographic meant to facilitate the viewer's understanding of the messages were used throughout the infographic. Both words and images were created based on Chinese culture. The draft in Chinese language was analyzed by the team and included both English and Chinese knowledgeable speakers to verify effectiveness of the images and how well the information could be understood by Chinese health professionals and the public. Next, the infographic was sent to faculty who are working in the mental health profession at Capital Medical University, School of Nursing to review. Upon their review and positive feedback, any edits recommended were incorporated, and the final version of the infographic was approved by the grant team. In the light of the informational infographic, the CMUSON faculty nurse champion also developed extended Chinese text information for further explanation and education. These included: FASD terminology and acronyms, cause and prevention, prevalence, signs and symptoms, diagnosis, the main ways of intervention and treatment, and alcohol SBI.

\section{Results}

\subsection{The dissemination of infographic and text informa- tion}

\subsubsection{Posting and distributing paper-based copies of info- graphic}

The CMUSON faculty nurse champion/visiting scholar's university, Capital Medical University, located in Beijing, China, is one of the highest rated medical universities in China. It consists of 10 schools, 18 affiliated hospitals and 1 teaching institute. Among the 18 affiliated hospitals, three hospitals undertake the main teaching tasks of the Capital Medical University. The CMUSON faculty nurse champion chose 8 billboards in three affiliated hospitals and the main campus of Capital Medical University for posting the infographic information for one week. The locations in the three hospitals were readily visible to patients, medical students, nurses and doctors. Records indicated that chosen locations included 1,147 inpatient beds/1,256 inpatient beds/1,401 beds with 1,366 nurses/1,412 nurses/1,390 nurses in addition to $5,000-8,000$ average daily out patients each as well as 7,000 medical students. The University-campus locations chosen reflected nursing and other medical students the most class traffic.

\subsubsection{Pushing electronic Chinese infographic and text in- formation through WeChat}

With the development of information technology (IT), social media, and smartphones for new information acquisition and exchange channels and tools, currently there are widely used platforms available for the study of health information. ${ }^{[15]}$ According to the information-motivation-behavior skills (IMB) model, information can be converted into action, and can inspire individuals and ultimately influence their attitudes and behavior. ${ }^{[10]}$ Studies have confirmed that mobile technology interventions significantly improved an array of health care outcomes such as self-management behavior in Chinese populations. ${ }^{[16]}$ WeChat is one of the most popular social media platforms worldwide, with more than 0.54 billion users in China and worldwide. It offers a free instant messaging application for Smartphones that enable the exchange of voice, text, pictures, videos, and location information via mobile phone indexes. WeChat has been proven to be an effective, sustainable, feasible, and well accepted strategy for improving and advancing health information awareness among the Chinese. ${ }^{[17]}$

The present project is based on one of WeChat's public accounts called WeChat Subscription Account, which is a type of We-Media service on the WeChat platform that can be freely acquired and used by individuals to disseminate information for internal communications to its audience on a daily base. Individuals can read messages and communicate with others via these Subscription accounts. Three days after the information was promoted, 1,616 individuals read the infographic and extended text information 1,848 times. This response included 1,238 (76.61\%) women, 363 $(22.46 \%)$ men, with $(15,0.93 \%)$ of unknown gender. The individuals represented 30 provinces of China. The number of readers has been rising daily with the CMUSON faculty nurse champion's promotion efforts.

\section{Discussion}

\subsection{The need for knowledge on FASD in China}

According to the World Health Organization Global Status Report on Alcohol and Health 2014, China represents high alcohol production and consumption. ${ }^{[9]} \mathrm{Li}$, et al. reported that $15 \%$ of Chinese women and $55.6 \%$ Chinese men drank alcohol. ${ }^{[11]}$ Although women were traditionally discouraged from drinking alcohol, the number of women who drink alcohol, especially the young women who are of childbearing age, has been rising dramatically with the fast growth of Chinese economy and the culture globalization. ${ }^{[4]}$ However, 
according to Wang's systematic review on FASD in China, FASD cases have been rarely reported ${ }^{[4]}$ which might be a result of a lack of knowledge of FASD for medical professionals leading to difficulty in diagnosing and recording FASD. Therefore, to eliminate this discrepancy, it is imperative to provide appropriate education regarding alcohol consumption and its negative consequences to both health professionals and the Chinese public population. Clearly, high alcohol consumption and the lack of information about its negative effects on fetal development makes this country a prime candidate for the role of nurse champions in promoting the awareness of FASD.

\subsection{Nursing faculty champions}

The nursing profession, the largest and most trusted sector of the healthcare workforce in China, is in a unique position to promote the awareness of basic knowledge of FASD for both the population of childbearing women and fellow healthcare professionals. Nurses are responsible for taking care of patients and providing care and education to the communities they serve; this includes the important role they have in the care of people who consume substances such as alcohol. ${ }^{[18,19]}$ It is critical that educational materials, such as the CMUSON Faculty Nurse Champion developed, including the infographic and the extended text information, are accessible not only to nursing students, but also to nurses and all medical staff to ensure that the philosophy of patient-centered care is provided via interprofessional and collaborative work. ${ }^{[20]}$

Because a champion is a "charismatic advocate of a belief, practice, program, policy and/or technology", ${ }^{[21]}$ nursing faculty can be invaluable champion resources to gain and sustain the goals of promoting the awareness of FASD both for Chinese health providers and the public. The CMUSON Faculty Nurse Champion designed, disseminated, and distributed Mandarin Chinese FASD infographic, leaflets, and WeChat public information to impact thousands of Chinese health providers and the general public to increase awareness of FASD and its cause.

\section{Conclusions}

There is a lack of knowledge regarding FASD in China. The nursing profession can change this by disseminating FASD information. Using a Nurse Faculty Champion is a logical, practical method to promote the awareness of FASD in China. One champion's efforts represent a beginning. Next steps include encouraging more nursing faculty to become champions, developing nursing FASD curricula, and increasing attention to FASD research initiatives.

\section{ACKNOWLEDGEMENTS}

This project was supported in part by funds from the U.S. Department of Health and Human Services, Centers for Disease Control and Prevention, under grant number 1U84DD001135. The information or content and conclusions are those of the author and should not be construed as the official position or policy of, nor should any endorsements be inferred by the CDC, DHHS, or the U.S. Government.

\section{CONFliCtS OF INTEREST Disclosure}

The authors have no conflicts of interest to report.

\section{REFERENCES}

[1] Center for Disease Control and Prevention. Fetal alcohol spectrum disorders (FASDs). 2015. Available from: https://www.cdc.go v/ncbddd/fasd/facts.html

[2] Gallup. Honesty/Ethics in Professions. Available from: http://www.gallup.com/poll/1654/honesty-ethic s-professions.aspx

[3] Williams JF, Smith VC. Fetal Alcohol Spectrum Disorders. Pediatrics. 2015; 136(5): e1395-1406. https://doi.org/10.1542/peds. 2 015-3113

[4] Wang YY, D’Amvailableato RC. Understanding Fetal Alcohol Spectrum Disorders in China. Journal of Pediatric Neuropsychology. 2017 https://doi.org/10.1007/s40817-017-0029-0

[5] Hoyme HE, Kalberg WO, Elliott AJ, et al. Updated Clinical Guidelines for Diagnosing Fetal Alcohol Spectrum Disorders. Pediatrics. 2016; 138(2). https ://doi.org/10.1542/peds . 2015-4256

[6] Hagan JF, Balachova T, Bertrand J, et al. Neurobehavioral Disorder Associated With Prenatal Alcohol Exposure. Pediatrics. 2016; 138(4). https://doi.org/10.1542/peds.2015-1553
[7] Warren KR, Hewitt BG. Fetal alcohol spectrum disorders: when science, medicine, public policy, and laws collide. Dev Disabil Res Rev. 2009; 15(3): 170-175. https://doi.org/10.1002/ddrr.71

[8] Tang YL, Xiang XJ, Wang XY, et al. Alcohol and alcohol-related harm in China: policy changes needed. Bull World Health Organ. 2013; 91(4): 270-276. https://doi.org/10.2471/BLT.12.107 318

[9] World Health Organization. Global status report on alcohol and health 2014. 2014. Available from: http://www. who.int/substance abuse/publications/global_alcohol_report/en/

[10] Li W, Han LQ, Guo YJ, et al. Using WeChat official accounts to improve malaria health literacy among Chinese expatriates in Niger: an intervention study. Malar J. 2016; 15(1): 567. https: //doi.org/10.1186/s12936-016-1621-y

[11] Li Y, Jiang Y, Zhang M, et al. Drinking behavior among men and women in China: the 2007 China Chronic Disease and Risk Factor Surveillance. Addiction. 2011; 106(11): 1946-1956. https: //doi.org/10.1111/j.1360-0443.2011.03514.x

[12] Ren A, Zhang L, Hao L, et al. Comparison of blood folate levels 
among pregnant Chinese women in areas with high and low prevalence of neural tube defects. Public Health Nutr. 2007; 10(8): 762768. https://doi.org/10.1017/S1368980007246786

[13] Roozen S, Black D, Peters GY, et al. Fetal Alcohol Spectrum Disorders (FASD): an Approach to Effective Prevention. Curr Dev Disord Rep. 2016; 3(4): 229-234. https ://doi.org/10.1007/s40474 -016-0101-y

[14] Babor TF, Higgins-Biddle JC, Higgins PS, et al. Training medical providers to conduct alcohol screening and brief interventions. Subst Abus. 2004; 25(1): 17-26. https://doi.org/10.1300/J465v2 5n01_04

[15] Forrest JI, Wiens M, Kanters S, et al. Mobile healthapplications for HIV prevention and care in Africa. Curr Opin HIV AIDS. 2015; 10(6): 464-471. PMid:26352394 https ://doi .org/10.1097/C0 H. 0000000000000198

[16] Corpman DW. Mobile health in China: a review of research and programs in medical care, health education, and public health. J Health Commun. 2013; 18(11): 1345-1367. https://doi.org/10.108 $0 / 10810730.2013 .778370$
[17] Kang X, Zhao L, Leung F, et al. Delivery of Instructions via Mobile Social Media App Increases Quality of Bowel Preparation. Clin Gastroenterol Hepatol. 2016; 14(3): 429-435, e423. https: //doi.org/10.1016/j.cgh.2015.09.038

[18] Pillon SC, Luis MAV, Laranjeira R. Nurses' training on dealing with alcohol and drug abuse: a question of necessity. Revista do Hospital das Clínicas. 2003; 58(2): 119-124. PMid:12845366 https://doi.org/10.1590/S0041-87812003000200011

[19] Barrett J, Jansen M, Cooper A, et al. Embracing a Nurse-Driven Alcohol Withdrawal Protocol through Quality Improvement. J Addict Nurs. 2017; 27(4): 234-240. https://doi .org/10.1097/JA N. 0000000000000142

[20] Kelley A, Aston L. An evaluation of using champions to enhance inter-professional learning in the practice setting. Nurse Education in Practice. 2011; 11(1): 35-40. https://doi.org/10.1016/j.ne pr.2010.06.003

[21] Howella JM, Sheab CM, Higgins CA. Champions of product innovations: defining, developing, and validating a measure of champion behavior. Journal of Business Venturing. 2005; 20(5): 641-661. https://doi.org/10.1016/j.jbusvent. 2004.06.001 